\title{
The robustness of acoustic analogies
}

\author{
By J. B. Freund $\dagger$, S. K. Lele AND M. Wei $\dagger$
}

Acoustic analogies for the prediction of flow noise are exact rearrangements of the flow equations $\mathcal{N}(\vec{q})=0$ into a nominal sound source $\mathcal{S}(\vec{q})$ and sound propagation operator $\mathcal{L}$ such that $\mathcal{L} \vec{q}=S(\vec{q})$. In practice, the sound source is typically modeled and the propagation operator inverted to make predictions. Since the rearrangement is exact, any sufficiently accurate model of the source will yield the correct sound, so other factors must determine the merits of any particular formulation. Using data from a twodimensional mixing layer direct numerical simulation (DNS), we evaluate the robustness of two analogy formulations to different errors intentionally introduced into the source. The motivation is that since $\mathcal{S}$ can not be perfectly modeled, analogies that are less sensitive to errors in $\mathcal{S}$ are preferable. Our assessment is made within the framework of Goldstein's generalized acoustic analogy, in which different choices of a base flow used in constructing $\mathcal{L}$ give different sources $\mathcal{S}$ and thus different analogies. A uniform base flow yields a Lighthill-like analogy, which we evaluate against a formulation in which the base flow is the actual mean flow of the DNS. The more complex mean flow formulation is found to be significantly more robust to errors in the energetic turbulent fluctuations, but its advantage is less pronounced when errors are made in the smaller scales.

\section{Introduction}

Jet noise remains a major contributing component of aircraft noise, especially at takeoff, and is especially challenging to reduce without decreasing jet exit velocities. Design tools with sufficient reliability to explore configurations (e.g. nozzle geometries) significantly different from those against which modeling parameters were tuned are lacking. Thus, the industry currently relies in part on an expensive Edisonian trial-and-error approach for seeking improvements. The principal difficulty is that the noise radiated by a jet depends in a complex way upon the details of the unsteady flow turbulence, which makes it harder to predict than the simpler mean statistics typically targeted by turbulence models.

Most approaches to predicting jet noise have three elements: designation of a noise source and propagation operator, modeling of the source, and solution of the radiated sound. Just as there is no clear separation of sound from the rest of the flow in nonlinear compressible turbulence, there is also no unique designation of a noise source in such a flow. Near flow Mach number $M$ of unity, which is where jets on civilian aircraft typically operate, many of the length and time scale separations common in acoustic analysis are unavailable to assist this designation. As a result, there are numerous possible choices for decomposing the flow equations, written compactly here for flow field $\vec{q}$ as $\mathcal{N}(\vec{q})=0$, into a nominal noise source $\mathcal{S}$ and propagation $\mathcal{L}$ operator: $\mathcal{L} \vec{q}=\mathcal{S}(\vec{q})$. This procedure is known as acoustic analogy; the rearrangement is exact, but $\mathcal{S}(\vec{q})$ is treated analogously to an externally applied noise source. The (usually linear) wave operator $\mathcal{L}$ provides the means to compute $\vec{q}$ in the far field which is the radiated noise.

$\dagger$ Theoretical and Applied Mechanics, University of Illinois at Urbana-Champaign 
Lighthill (1952) introduced the acoustic analogy framework, selecting $\mathcal{L}$ to be a homogeneousmedium scalar wave operator acting on the density perturbation $\rho^{\prime}$,

$$
\mathcal{L} \vec{q}=\frac{\partial^{2} \rho^{\prime}}{\partial t^{2}}-a_{\infty}^{2} \nabla^{2} \rho^{\prime}=\mathcal{S}(\vec{q})
$$

with the source

$$
\mathcal{S}(\vec{q})=\frac{\partial^{2}}{\partial x_{i} \partial x_{j}}\left(\rho v_{i} v_{j}+\left(p^{\prime}-a_{\infty}^{2} \rho^{\prime}\right) \delta_{i j}-\tau_{i j}\right)
$$

where $a_{\infty}$ is the constant ambient speed of sound, $\rho^{\prime}=\rho-\rho_{\infty}$ is density perturbation, $p^{\prime}=p-p_{\infty}$ is pressure perturbation, and $v_{i}$ are velocity components. $\dagger$ Inversion of the wave operator is trivial in this case, but modeling the source in absence of precise space-time near-field information is not.

Lighthill recognized from the beginning (Lighthill 1952) that his decomposition was in a sense artificial. Since the propagation operator $\mathcal{L}$ in (1.1) does not redirect sound, refraction is effectively lumped into $\mathcal{S}$ in (1.2) and is thus indistinguishable from a true noise source. The separation of propagation and generation has troubled the aeroacoustic community ever since. It can be argued that $\mathcal{L}$ and $\mathcal{S}$ that better separate true sources from propagation provide a firmer platform upon which to begin modeling the sources. The linearized Lilley equation (Lilley 1974; Goldstein 1976) is a common choice for this. In this formulation, a parallel base flow and any refraction it causes is explicit in $\mathcal{L}$. However, it does not provide any obvious simplification of the source $\mathcal{S}$, still leaving complex combinations of terms (e.g. Colonius et al. 1997) which must be modeled in making predictions. Since there is no clear simplification that comes about by including more propagation physics into $\mathcal{L}$, it can therefore also be argued that the Lighthill approach is no worse off than such a more sophisticated approach. Since acoustic analogies are exact, an accurate representation of the source in the simpler Lighthill analogy provides the correct sound despite the multiple physical effects grouped into it. Which approach is best can be argued back and fourth, but since different acoustic analogies require different source models, it is difficult to make clear head-to-head comparisons between them and thus difficult to judge if one is preferable to others. Also, since the source is complex, there are typically sufficient free parameters to make it match data for any particular jet.

In this paper, we take a new approach and empirically assess the robustness of different acoustic analogies to modeling errors in their respective $\mathcal{S}$ 's. Since $\mathcal{S}$ will never be modeled exactly in practice, we explore whether some $\mathcal{L} \vec{q}=\mathcal{S}(\vec{q})$ decompositions are more robust to errors than others. It is known, for example, that small errors in the source for the Lilley equation can lead to large (order of magnitude) errors in the noise predicted (Colonius et al. 1997). To make our assessment, we employ Goldstein's recently developed generalized acoustic analogy, which provides a single clear framework for a family of consistent $\mathcal{L}$ 's and $\mathcal{S}$ 's. The formulation is described in section 2.1. The noise source data are taken from a direct numerical simulation (DNS) described in section 2.2, with errors of types purposely introduced as described in section 2.3. Specifically, we consider Lighthill's homogeneous-medium wave equation approach and a formulation in which $\mathcal{L}$ includes the exact mean flow taken from the DNS.

$\dagger$ This form of Lighthill's equation is equivalent to his formulation and more convenient. 


\section{Preliminaries}

\subsection{Formulation}

The propagation operator in Goldstein's (2003) generalized acoustic analogy is similar to linearized Euler equations:

$$
\begin{gathered}
\bar{\rho} \frac{\bar{D}}{D t} \frac{\rho^{\prime}}{\bar{\rho}}+\frac{\partial}{\partial x_{j}} \bar{\rho} u_{j}^{\prime}=0 \\
\bar{\rho}\left(\frac{\bar{D}}{D t} u_{i}^{\prime}+u_{j}^{\prime} \frac{\partial \tilde{v}_{i}}{\partial x_{j}}\right)+\frac{\partial p_{e}^{\prime}}{\partial x_{i}}-\rho^{\prime} / \bar{\rho} \frac{\partial \tilde{\tau}_{i j}}{\partial x_{j}}=\frac{\partial}{\partial x_{j}}\left(e_{i j}^{\prime}-\tilde{e}_{i j}\right) \\
\frac{1}{\gamma-1}\left(\frac{\bar{D} p_{e}^{\prime}}{D t}+\gamma p_{e}^{\prime} \frac{\partial \tilde{v}_{j}}{\partial x_{j}}+\gamma \frac{\partial}{\partial x_{j}} \bar{p} u_{j}^{\prime}\right)-u_{i}^{\prime} \frac{\partial \tilde{\tau}_{i j}}{\partial x_{j}}=\frac{\partial}{\partial x_{j}}\left(\eta_{j}^{\prime}-\tilde{\eta}_{j}\right)+\left(e_{i j}^{\prime}-\tilde{e}_{i j}\right) \frac{\partial \tilde{v}_{i}}{\partial x_{j}}
\end{gathered}
$$

where

$$
\frac{\bar{D}}{D t} \equiv \frac{\partial}{\partial t}+\tilde{v}_{j} \frac{\partial}{\partial x_{j}}
$$

and

$$
\tilde{\tau}_{i j} \equiv \delta_{i j} \bar{p}-\tilde{T}_{i j}-\tilde{\sigma}_{i j}
$$

The base flow which defines the specific analogy has density $\bar{\rho}$, pressure $\bar{p}$, and velocity $\tilde{v}_{i}$ and satisfies exact equations with sources $\tilde{T}_{i j}, \tilde{H}_{i j}$ and $\tilde{H}_{0}$ (Goldstein 2003). For example, the governing equations for a time-averaged base flow has Reynolds stresses as sources. Perturbations from this base flow are $\rho^{\prime}, p^{\prime}$, and $v_{i}^{\prime}$. To put (2.1) into its relatively clean form, new dependent variables were constructed from nonlinear combinations of the primitive variables as

$$
p_{e}^{\prime} \equiv p^{\prime}+\frac{\gamma-1}{2} \rho v_{i} v_{i}+(\gamma-1) \tilde{H}_{0} \quad \text { and } \quad u_{i}^{\prime} \equiv \rho \frac{v_{i}^{\prime}}{\bar{\rho}},
$$

which become $p_{e}^{\prime}=p^{\prime}$ and $u_{i}=v_{i}$ in the linear acoustic limit.

The source terms on the right-hand side of $(2.1 b)$ and $(2.1 c)$ are the $\mathcal{S}$ discussed in section 1 . They are

$$
\begin{aligned}
e_{i j}^{\prime} & \equiv-\rho v_{i}^{\prime} v_{j}^{\prime}+\frac{\gamma-1}{2} \delta_{i j} \rho v_{k}^{\prime} v_{k}^{\prime}+\sigma_{i j}^{\prime} \\
\tilde{e}_{i j} & \equiv \tilde{T}_{i j}-\delta_{i j}(\gamma-1) \tilde{H}_{0} \\
\eta_{i}^{\prime} & \equiv-\rho v_{i}^{\prime} h_{0}^{\prime}-q_{i}^{\prime}+\sigma_{i j} v_{j}^{\prime} \\
\tilde{\eta}_{i} & \equiv \tilde{H}_{i}-\tilde{T}_{i j} \tilde{v}_{j},
\end{aligned}
$$

where $\sigma$ is a viscous stress tensor and $q$ is a diffusive heat flux. The enthalpy $h_{0}^{\prime}$ is $h_{0}^{\prime} \equiv h^{\prime}+v_{i}^{\prime} v_{i}^{\prime} / 2$, where $h^{\prime}$ is a perturbation to the base flow thermodynamic enthalpy.

We consider two base flow choices in this paper. The first is a uniform flow,

$$
\bar{\rho}=\rho_{\infty} \quad \bar{p}=p_{\infty} \quad \tilde{v}_{1}=V_{1} \quad \text { and } \quad \tilde{v}_{2}=\tilde{v}_{3}=0,
$$

which gives a Lighthill-like formulation for a uniform velocity homogeneous medium. $V_{1}$ is the velocity of the slow speed side of the mixing layer. In this case, $\tilde{T}_{i j}, \tilde{H}_{i j}$ and $\tilde{H}_{0}$ 
are all zero, so the sources are

$$
e_{i j}^{\prime}-\tilde{e}_{i j}=-\rho v_{i}^{\prime} v_{j}^{\prime}+\frac{\gamma-1}{2} \delta_{i j} \rho v_{k}^{\prime} v_{k}^{\prime}
$$

and

$$
\eta_{i}^{\prime}-\tilde{\eta}_{i}=-\rho v_{i}^{\prime} h_{0}^{\prime},
$$

where we have omitted molecular diffusion terms as we do throughout in the discussion and in the numerical calculations. In this case, perturbations are from the uniform flow (2.6), not the mean flow.

In the second case, we use the time averaged DNS flow to be the base flow. An overbar $\left(^{-}\right)$indicates a time average with corresponding Favre average $\left({ }^{*}\right)$. Thus we have noise sources

$$
e_{i j}^{\prime}-\tilde{e}_{i j}=-\rho v_{i}^{\prime} v_{j}^{\prime}+\overline{\rho v_{i}^{\prime} v_{j}^{\prime}}+\frac{\gamma-1}{2} \delta_{i j}\left(\rho v_{k}^{\prime} v_{k}^{\prime}-\overline{\rho v_{k}^{\prime} v_{k}^{\prime}}\right)
$$

and

$$
\eta_{i}^{\prime}-\tilde{\eta}_{i}=-\rho v_{i}^{\prime} h_{0}^{\prime}+\overline{\rho v_{i}^{\prime} h_{0}^{\prime}}
$$

which have zero mean.

\subsection{DNS Data and Acoustic Analogy Solution}

We will study the robustness of these two acoustic analogies for a two-dimensional mixing layer flow between Mach 0.9 and Mach 0.2 free streams of the same temperature. The instantaneous vorticity is visualized in figure 1 , which also shows that the physical domain of the simulation extends from $x=0$ to $200 \delta$ downstream and from $y=-80 \delta$ to $80 \delta$ in the transverse direction, where $\delta$ is the vorticity thickness at the inflow boundary. The Reynolds number based on the velocity difference and $\delta$ is 500 . The physical domain was surrounded by a boundary zone on all sides to absorb outgoing disturbance. The flow was excited by low amplitude random forcing applied in the inflow boundary zone at 8 frequencies randomly distributed between zero and twice the most unstable frequency predicted by linear stability analysis. To simulate the flow, the compressible flow equations were solved using high-order finite differences and fourth-order Runge-Kutta time advancement and without modeling approximations. Full details of the numerical method and simulation procedure, including the specifics of the inflow excitation, are provided elsewhere (Wei \& Freund 2004). The only difference here is that the present $x$ domain is twice as long as that considered in those mixing layer control studies and the simulation time is doubled.

Flow fields were saved every four numerical time steps during simulation. All necessary averages, correlations, and source terms needed for the acoustic analogies were computed directly from this stored DNS data. The left-hand sides of (2.1) were solved directly on the same mesh as the direct numerical simulation using a similar algorithm with the same numerical time step as the DNS. The source was interpolated in time as necessary using a cubic spline with 16 knots. For both the DNS data and the analogy solutions, the sound was extrapolated into the far-field by a semi-analytic solution of the wave equation in the same manner as by Freund (2001). The full details of this procedure are also documented elsewhere (Wei \& Freund 2004). 


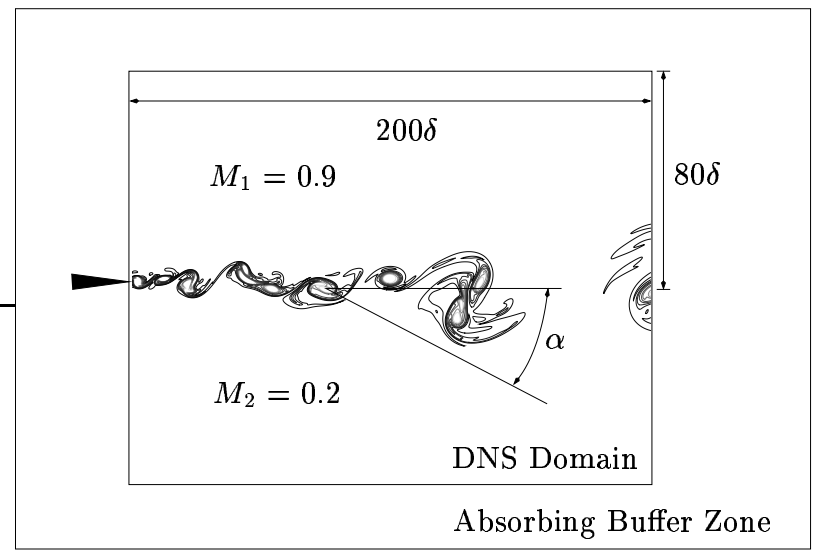

FIGURE 1. Schematic of the computational domain with vorticity magnitude showing the mixing layer.

\subsection{Source Errors}

As discussed in section 1, the primary objective of this work is to assess the robustness of the noise predictions to errors in the nominal noise source, so we must somehow specify these errors. For now, we only consider errors that arise from omitting part of the flow solution from the source computation. In practice, this error might be the missing small-scale turbulence in a large-eddy simulation or due to imperfect modeling of the larger turbulent scale in, say, a parabolized Navier-Stokes model of a mixing layer or jet (Bertolotti \& Colonius 2003; Chueng \& Lele 2004), but we make no attempt to rigorously represent to any particular source of error. Instead, the flow was simply decomposed into empirical Karhunen-Loeve (KL) eigenfunctions $\vec{\psi}_{i}(\mathbf{x})$ (Holmes, Lumley \& Berkooz, 1996), also commonly called Proper Orthogonal Decomposition or POD modes, with time coefficients $a_{i}(t)$ such that the flow $\vec{q}$ is reconstructed as

$$
\vec{q}(\mathbf{x}, t)=\sum_{i=1}^{N} a_{i}(t) \vec{\psi}_{i}(\mathbf{x}) .
$$

In this study, the kinetic energy norm

$$
E=\int_{\mathcal{V}} \rho u_{i} u_{i} d \mathcal{V}
$$

defines the eigenfunctions in the usual way. Subsets of these modes were used to reconstruct flow and calculate approximations to $e_{i j}$ and $\eta_{i}$ defined in (2.5). To compute $\vec{\psi}_{i}$, we used the method of snapshots (Sirovich, 1987) with 558 data fields sampled at regular intervals from the 3907 available fields stored from the simulation. The resulting KL modes are used only to define a spectral decomposition of the flow which has attractive properties for our purposes, so special significance is given to any particular modes. An alternative approach to introducing error would be to specify length and time scales at which to filter the data. While that approach might better connect the present errors to the class of errors expected in a large-eddy simulation, it requires that several filtering parameters be specified and would not provide a single framework for all the types of error we wish to introduce. The empirical eigenfunctions on the other hand follow directly from the definition of the energy norm (2.12). The modes in approximately homogeneous 


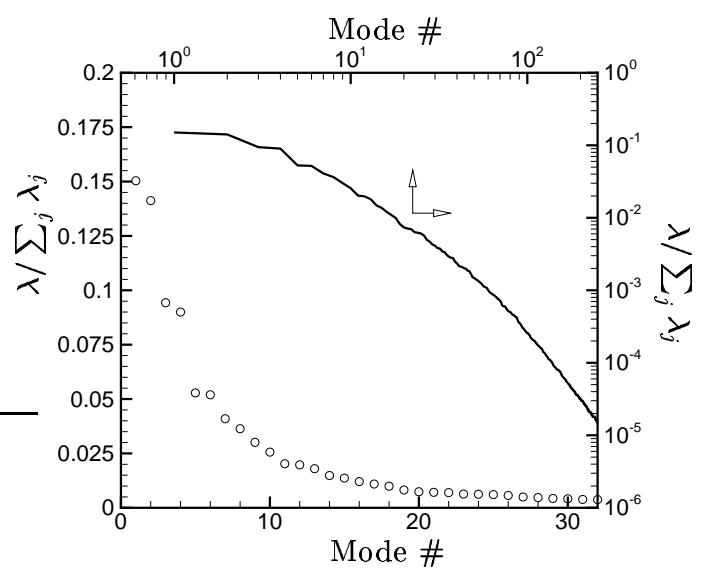

FIGURE 2. Fraction of kinetic energy defined by (2.12) of each mode. The — line corresponds to the top and right logarithmic axes.

flows, such as the streamwise coordinate direction in the present case, can be related to Fourier like modes via WKB analysis (Moser 1994).

The fraction of the total energy contained by each KL modes is shown in figure 2 . The lowest-order modes contain most of this energy and seem to come in pairs. Similar pairing has been observed even in turbulent jets (Freund \& Colonius 2002) and is necessitated by the advection of slowly evolving structures downstream. The pairs appear as $\pi / 2$ phase shifted WKB-like wave packets in $x$ which fit together like sine's and cosine's. The higher-order modes have the exponential energy decay expected for any such spectral representation of a low-Reynolds-number flow. Figure 3 shows the spatial form of the $y$-velocity component of selected modes and their corresponding time coefficients $a_{i}(t)$. Clearly, the lower-order modes are larger scale and lower frequency.

We consider two types of erroneous reconstruction for our robustness assessment. The first is simple truncation of the reconstruction series in (2.11) as

$$
\vec{q}(\mathbf{x}, t)=\sum_{i=1}^{N_{t}} a_{i}(t) \vec{\psi}_{i}(\mathbf{x}),
$$

where $N_{t}$ is less than the total number of 558 modes available. Specifically, we consider cases with $N_{t}=16,32$, and 128 , which represent $81.2,91.3$, and 99.3 percent of the energy, respectively. The other error we introduce is a reduction in the coefficient of a low-order mode. The flow field in this case is reconstructed by (2.13) with $N_{t}=128$, but with $a_{i}$ reduced for selected small $i$. In particular, we take $a_{1}=0.5 a_{1}$ and $a_{3}=0.5 a_{3}$.

\section{Results}

Table 1 describes the six cases for which we present results. Sound spectra for all these cases are presented in figures 4 to 6 at directivity angles $\alpha=40^{\circ}, 90^{\circ}$, and $140^{\circ}$, where $\alpha$ is measured from the downstream $x$ axis toward the slow speed stream (see figure 1). At all angles the full source case (a) produces nearly a perfect match with the DNS directivity, as it should if implemented correctly. The small differences are believed to be due to boundary conditions and other numerical errors and are much smaller than the disagreement we will see in the other cases. 

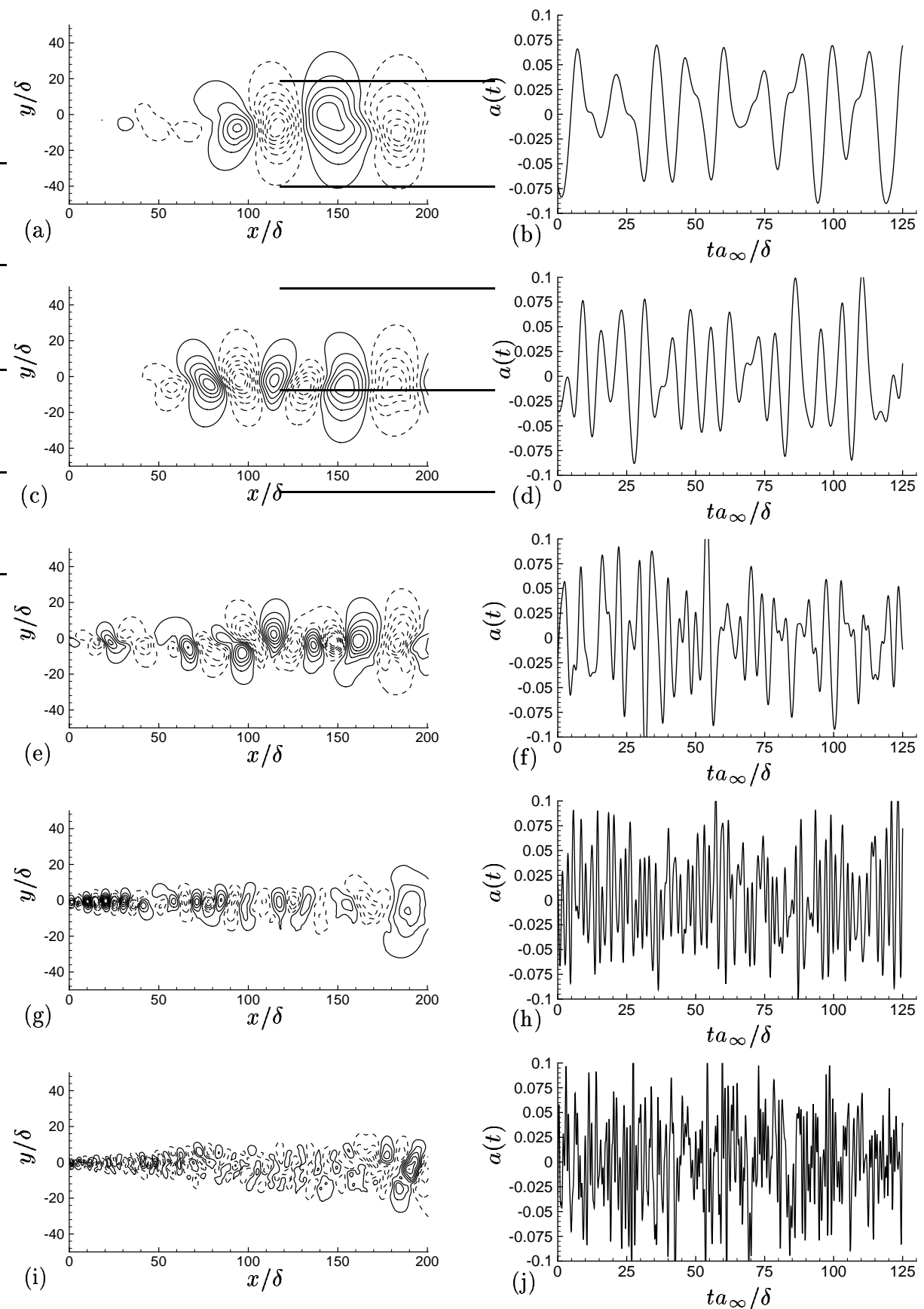

FiguRE 3. Mode shape and time coefficients for selected modes: (a-b) 1, (c-d) 3, (e-f) 16, (g-h) 32 , and (i-j) 128.

Despite representing nearly all the kinetic energy of the mixing layer, retaining 128 KL modes (case b) for both cases produce significant errors in the higher frequencies, especially at $\alpha=90^{\circ}$. The mean-flow analogy is slightly closer to the DNS at $\alpha=40^{\circ}$, but 


\begin{tabular}{ccl} 
Case & Energy Retained & \multicolumn{1}{c}{ Description } \\
a & $100.0 \%$ & Full souce information \\
b & $99.3 \%$ & 128 modes \\
c & $91.3 \%$ & 32 modes \\
d & $81.2 \%$ & 16 modes \\
e & $92.5 \%$ & Modes with $a_{1}=a_{1} / 2$ \\
f & $95.3 \%$ & Modes with $a_{3}=a_{3} / 2$
\end{tabular}

TABle 1. Cases presented.

otherwise the errors are effectively the same, giving neither formulation a clear advantage. Both make the noise too intense at these high frequencies indicating that the error is due to a disruption of cancellations that occur with the full source. Retaining fewer modes as in cases (c) and (d) leads to similar errors, but at lower and lower frequencies, which is in accord with our observation that the lower order modes have relatively lower frequencies. For the 16-mode case (d), the high frequencies are also under predicted, apparently because they are completely missing from the source in this case. The meanflow analogy performs slightly better, but does not show a strong advantage.

A much more significant difference is observed when we disrupt the lower frequency, larger-scale structures by altering the lowest-order KL modes. The (e) and (f) frames of figures 4 to 6 show these cases. Since modes only up through 128 were retained, we expect the same errors as in case (b) at high frequencies, but at $\alpha=40^{\circ}$ and $140^{\circ}$ we also now see large over prediction errors in the low frequencies for the uniform-flow (Lighthill) analogy. The mean-flow analogy case behaves significantly better. Clearly, the complexity introduced by better distinguishing propagation from true acoustic sources is worthwhile in this case.

\section{Discussion}

There are two ways that we can anticipate acoustic analogies being used in a time dependent formulation of the type tested here. The first would be in conjunction with a large-eddy simulation or similar accelerated solution of the flow equations which represents only the larger, more energetic scales of the flow. The analogy in this case might be used in conjunction with a sub-grid-scale source model to predict the noise from the missing scales (e.g. Bodony \& Lele 2003). The quantitative details of our assessment are not to be heavily relied upon in this case since the flow we have studied is two dimensional, though the mean flow itself is similar to that of a turbulent mixing layer so we do not anticipate any fundamental difference with that case. This application best corresponds to error models in which we truncated (2.11) sum at finite $N_{t}$, omitting for the most part smaller scale unsteadiness from the computation.

The second type of time dependent acoustic analogy application we envision would be in conjunction with a model for the largest scales of a turbulent flow, such as a solution of the parabolized Navier-Stokes equation (Bertolotti \& Colonius 2003; Chueng \& Lele 2004) or a dynamic model constructed by projecting KL modes onto the governing equations. Such a representation would, of course, contain some errors, which we modeled by altering the time coefficient of the lowest-order modes.

The results above suggest that neither formulation has a clear advantage for modeling the contribution of the missing small scales to the higher frequencies in the noise 

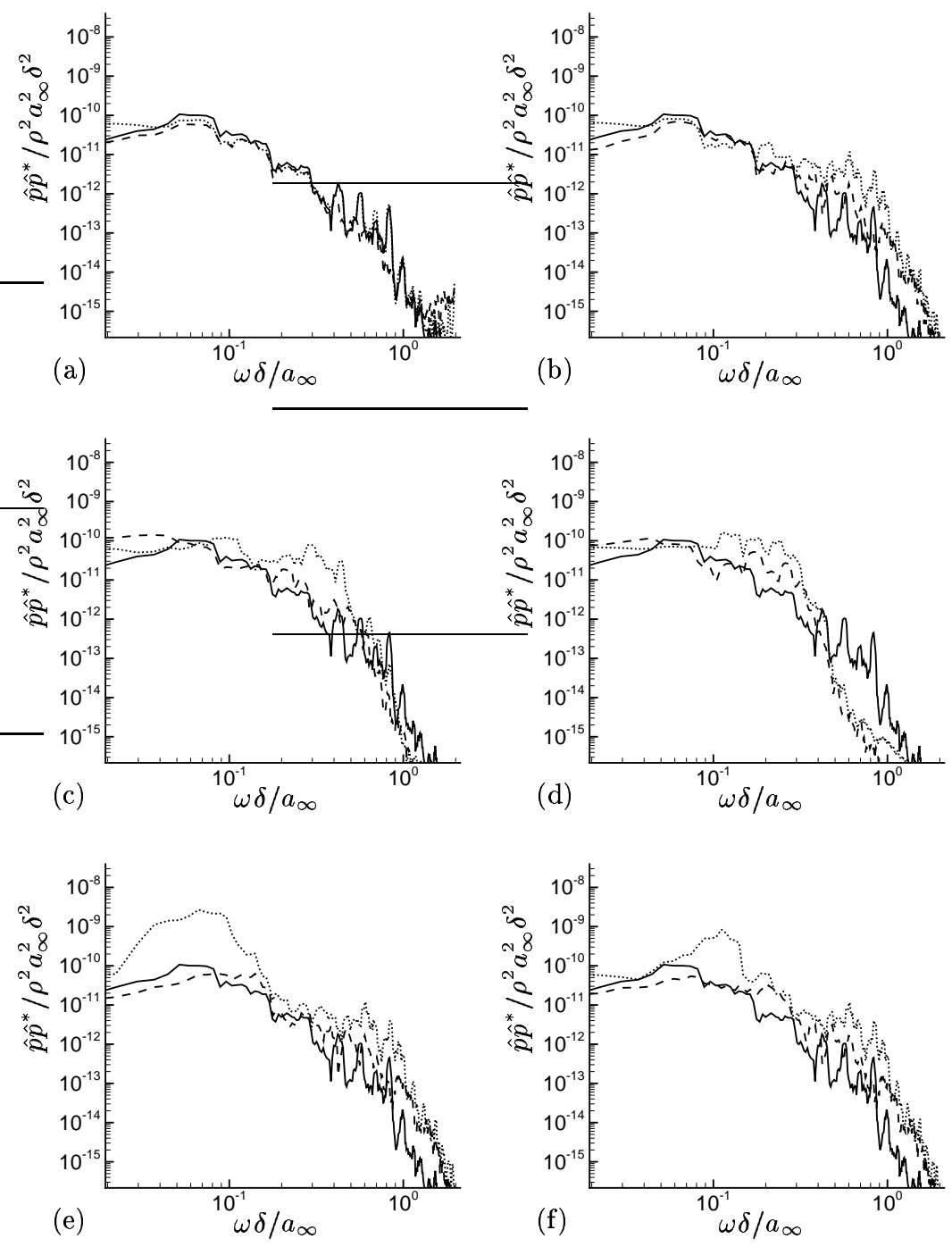

FigURE 4. Sound pressure spectra at $\alpha=40^{\circ}$ : — DNS; ---- DNS-mean base flow analogy; ........ uniform base flow (Lighthill) analogy. Frames (a) through (f) show cases a through $f$ as defined in table 1.

spectrum, though this might, of course, depend upon the specifics of the particular formulation used. However, the mean-flow analogy formulation does seem to be preferable in the second case, where the Lighthill-like formulation shows significant errors.

A purported disadvantage of the mean-flow analogy is that the resulting linearized Euler equations support instability wave homogeneous solutions, which have the potential to overwhelm the solution. Methods have been proposed for removing or reducing these (e.g. Bogey, Bailly \& Juvé 2002) but, as anticipated by Goldstein (2003), no special treatment was required in the present case. The homogeneous solution is excited, but does not degrade the acoustic predictions. 

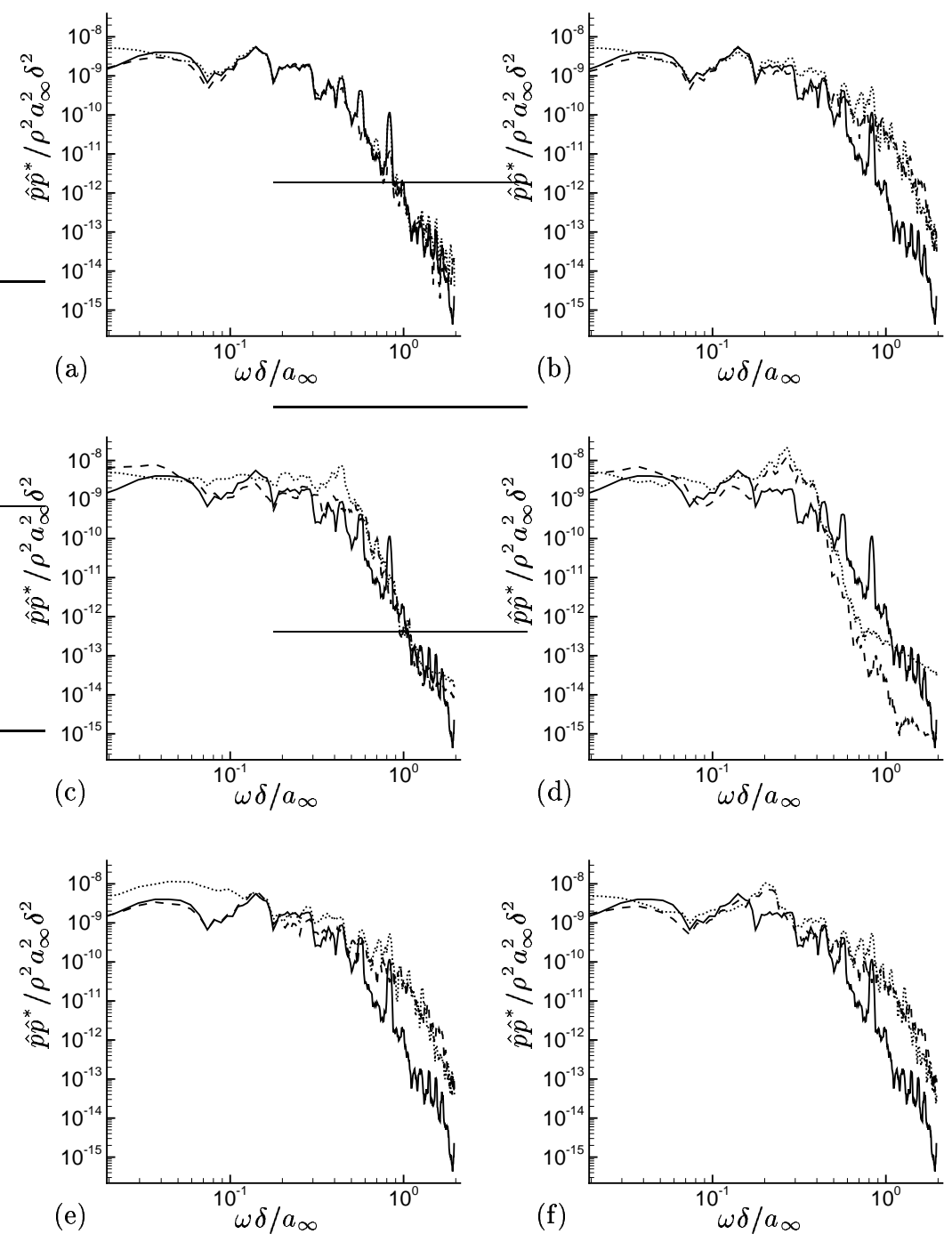

FiguRe 5. Same as figure 4 at $\alpha=90^{\circ}$.

\section{Conclusions}

The key conclusion is that formulating the acoustic analogy with the mean flow appearing in the propagation operator within Goldstein's (2003) formulation leads to a significantly more robust formula for the far-field sound. The errors we introduced into the low frequency, larger-scales do not in this case disproportionately affect the sound field. The same errors introduced into the source when the base flow is uniform, which corresponds to the Lighthill analogy, cause order-of-magnitude errors in the noise spectrum radiated to both upstream and downstream angles. The mean flow analogy is therefore more attractive by this measure for predicting noise when an imprecise dynamic model is used for the large-scale structures. However, both formulations show similar robustness when errors are introduced into the small-scale higher-frequency components of the 

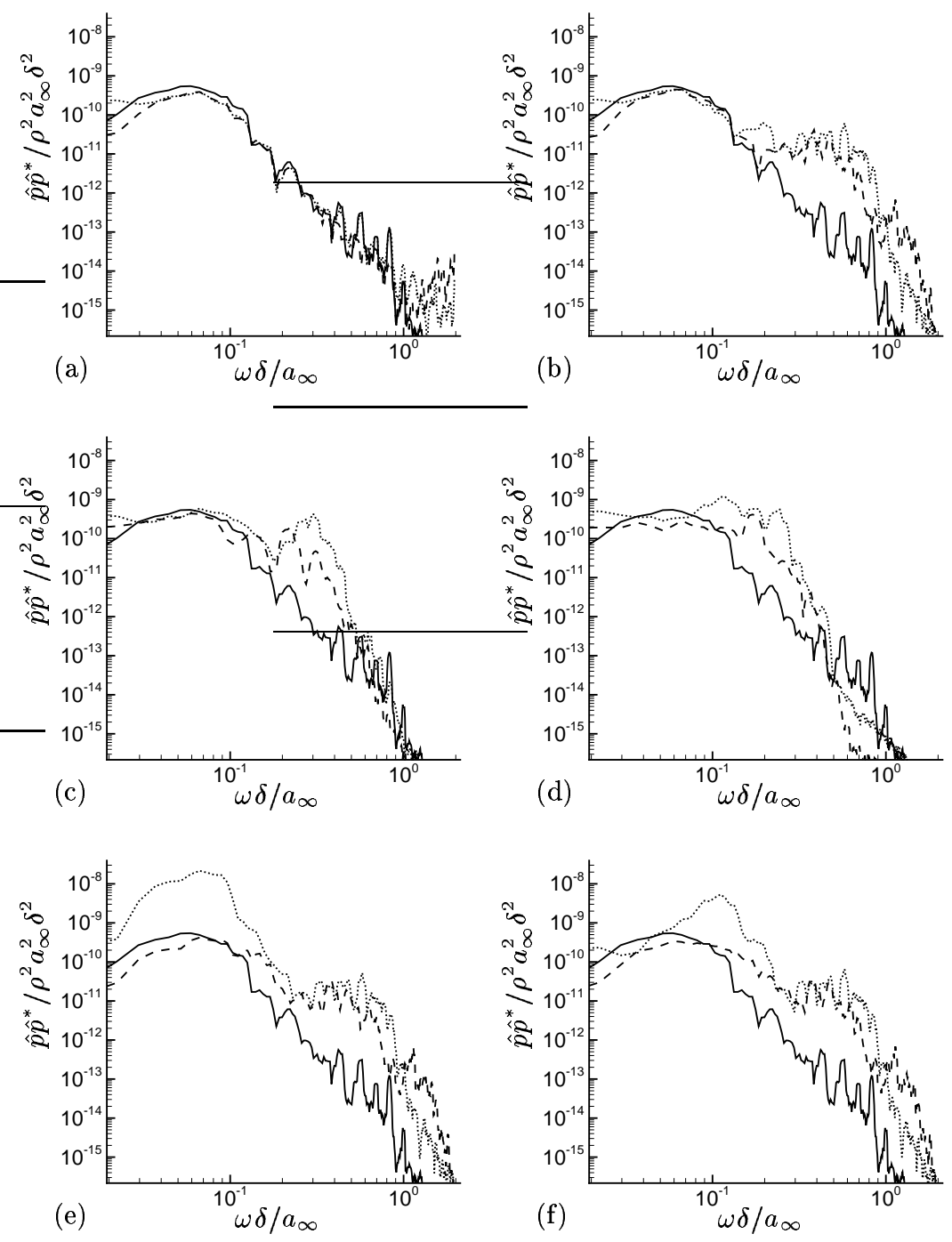

Figure 6. Same as figure 4 at $\alpha=140^{\circ}$.

source by our method, which by our measure indicates that neither has a clear advantage. Further testing with a turbulent flow is warranted.

JBF gratefully acknowledges support from NASA. The mixing layer DNS simulation were supported by AFOSR.

\section{REFERENCES}

Bertolotti, F. \& Colonius, T. 2003 On the noise generated by shear-layer instabilities in turbulent jets. AIAA Paper 2003-1062. 
BOdony, D. J. \& LeLE, S. K. 2003 A statistical subgrid scale noise model: formulation. AIAA Paper 2003-3252.

Bogey, C., Bailly, C. \& JuvÉ, D. 2002 Computation of flow noise using source terms in linearized Euler's equations. AIAA J. 40 (2), 235.

Cheung, L. \& Lele, S. 2004 Acoustic radiation from subsonic and supersonic mixing layers with nonlinear PSE. AIAA Paper 2004-0363.

Colonius, T., Lele, S. K. \& Moin, P. 1997 Sound generation in a mixing layer. $J$. Fluid Mech. 330, 375-409.

FReUnd, J. B. 2001 Noise sources in a low-Reynolds-number turbulent jet at Mach 0.9. J. Fluid Mech. 438, 277-305.

Freund, J. B. \& Colonius, T. 2002 POD analysis of sound generation by a turbulent jet. AIAA Paper 2002-0072.

Goldstein, M. E. 1976 Aeroacoustics. McGraw-Hill Book Co.

Goldstein, M. E. 2003 A generalized acoustic analogy. J. Fluid Mech. 488, 315-333.

Holmes, P., Lumley, J. L. \& Berkooz, G. 1996 Turbulence, Coherent Structures, Dynamical Systems and Symmetry. Cambridge: Cambridge University Press.

Lighthill, M. J. 1952 On sound generated aerodynamically: I. General theory. Proc. Royal Soc. Lond. A 211, 564-587.

Lilley, G. M. 1974 On the noise from jets. Tech. Rep. CP-131. AGARD.

Moser, R. D. 1994 Kolmogorov inertial range spectra for inhomogeneous turbulence. Phys. Fluids 6 (2), 794-801.

Sirovich, L. 1987 Chaotic dynamics of coherent structures. Parts I-III. Quarterly of Applied Math. XLV (3), 561-582.

Wei, M. \& Freund, J. B. 2004 A quiet free shear flow. submitted J. Fluid Mech. 